\title{
Interesting Findings of High-resolution Manometry Before and After Treatment in a Case of Diffuse Esophageal Spasm
}

\author{
Hee Man $\mathrm{Kim}^{1}$ and Tae Hee $\mathrm{Lee}^{2 *}$ \\ ${ }^{1}$ Department of Internal Medicine, Myongji Hospital, Kwandong University College of Medicine, Goyang, Gyeonggi-do, Korea; and ${ }^{2}$ Institute \\ for Digestive Research, Soonchunhyang College of Medicine, Seoul, Korea
}

A 45 -year-old woman visited the clinic presenting with squeezing chest pain during or after meal for 2 weeks. The findings of her esophagogastroduodenoscopy were unremarkable. Upon suspicion of non-erosive reflux disease, a proton pump inhibitor (pantoprazole $40 \mathrm{mg}$ ) had been prescribed to her, however her symptom was not relieved. She then was admitted to the hospital. To exclude coronary heart disease, cardiac tests including electrocardiography and echocardiography were performed, but no abnormality was found. To investigate esophageal motility disorder, high-resolution manometry (HRM) was performed, which revealed normal integrated relaxation pressure in $30 \%$ of swallows with reduced distal latency (DL, $<4.5 \mathrm{sec}$ ) and increased distal contractile integral (DCI) (Table and Figure A). Diffuse esophageal spasm (DES) was diagnosed and diltiazem $(180 \mathrm{mg})$ was additionally prescribed to her. After 10 days of treatment, her symptom was moderately relieved and the short-term follow-up HRM showed slightly improved DL and decreased DCI (Figure B). After 90 days, her symptom disappeared and HRM findings completely were normalized (Figure C).

According to the Chicago classification, DES is defined as normal mean integrated relaxation pressure and $\geq 20 \%$ of swallows with reduced DL $(<4.5 \mathrm{sec}){ }^{1}$ There are no large longi- tudinal studies of natural history in patients with DES. One small prospective study has reported that during long-term follow-up, the manometric findings of DES patients changed very little and the intensity of symptoms reduced. ${ }^{2,3}$ It suggests that acid sup-

Table. The Change of Symptoms and Manometry Findings Before and After Treatment

Baseline

After treatment

10 days 90 days

Chest pain

Frequency

Intensity $^{\mathrm{a}}$

Daily Weekly None

Dysphagia

Frequency

Intensity $^{a}$

Manometry

$\begin{array}{llll}\text { Integrated relaxation pressure }(\mathrm{mmHg}) & 3.2 & 3.9 & 2.5\end{array}$

$\begin{array}{llll}\text { Mean distal latency }(\mathrm{sec}) & 4.8 & 4.9 & 5.2\end{array}$

Frequency of reduced distal latency ${ }^{\mathrm{b}}(\%) \quad \begin{array}{llll}30 & 20 & 0\end{array}$

$\begin{array}{llll}\text { Mean distal contractile integral } & 6178.0 & 365.5 & 1393.3\end{array}$

$(\mathrm{mmHg} / \mathrm{cm} / \mathrm{sec})$

${ }^{\mathrm{a}}$ Visual analogue scale, ${ }^{\mathrm{b}}$ Reduced distal latency: $<4.5 \mathrm{sec}$.

Received: November 23, 2012 Revised: November 27, 2012 Accepted: November 27, 2012

(c) This is an Open Access article distributed under the terms of the Creative Commons Attribution Non-Commercial License (http://creativecommons. org/licenses/by-nc/3.0) which permits unrestricted non-commercial use, distribution, and reproduction in any medium, provided the original work is properly cited.

*Correspondence: Tae Hee Lee, MD

Institute for Digestive Research, Soonchunhyang College of Medicine, 59, Deasagwan-ro, Yongsan-gu, Seoul 140-743, Korea

Financial support: None.

Tel: +82-2-709-9691, Fax: +82-2-709-9696, E-mail: iman0825@schmc.ac.kr

Conflicts of interest: None. 

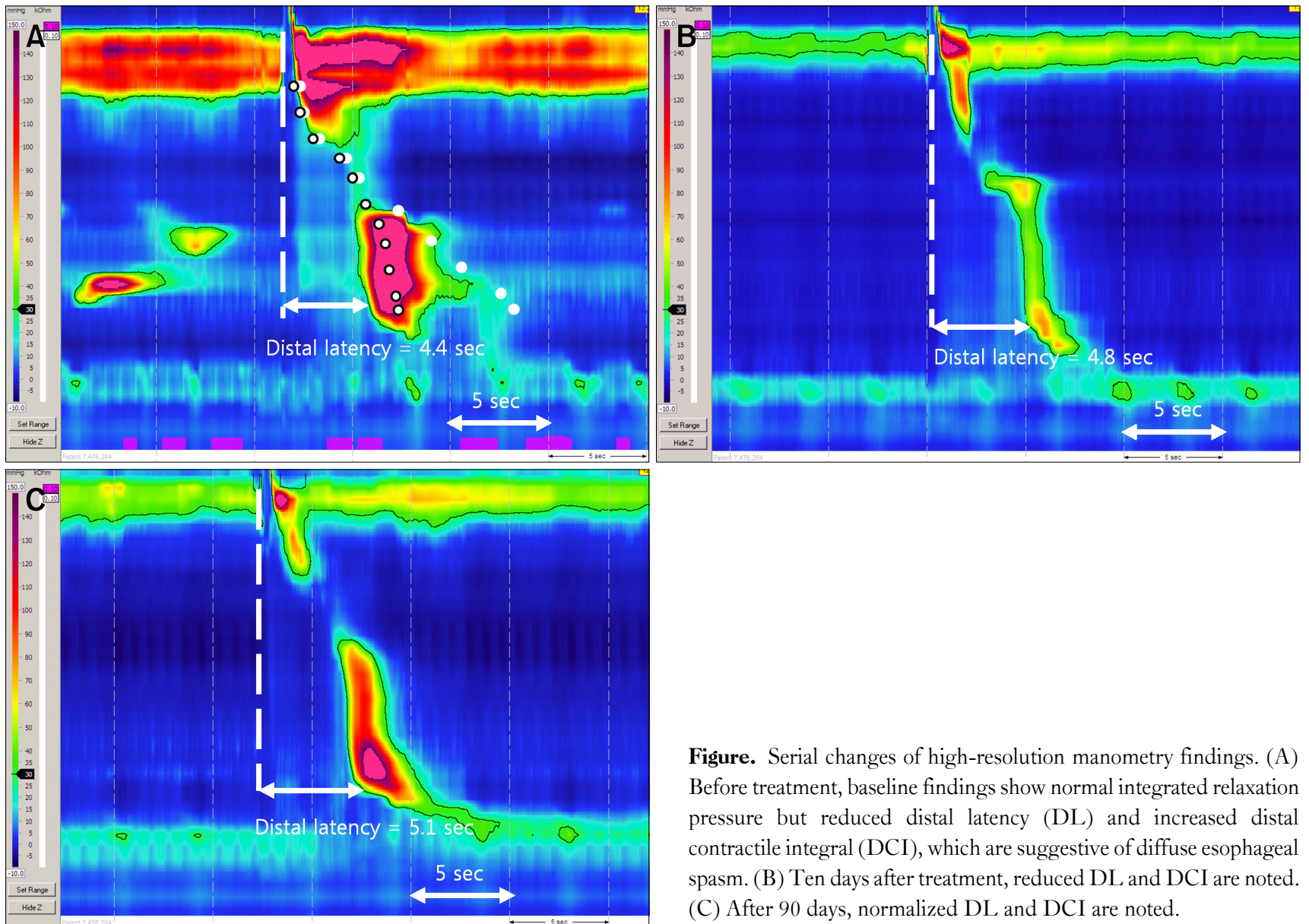

Figure. Serial changes of high-resolution manometry findings. (A) Before treatment, baseline findings show normal integrated relaxation pressure but reduced distal latency (DL) and increased distal contractile integral (DCI), which are suggestive of diffuse esophageal spasm. (B) Ten days after treatment, reduced DL and DCI are noted. (C) After 90 days, normalized DL and DCI are noted.

pression and smooth muscle relaxation are effective in some patients with DES. ${ }^{2}$ Herein is an interesting case of DES, in which both HRM features and symptoms responded to proton pump inhibitor and calcium channel blocker.

\section{References}

1. Bredenoord AJ, Fox M, Kahrilas PJ, et al. Chicago classification criteria of esophageal motility disorders defined in high resolution esophageal pressure topography. Neurogastroenterol Motil 2012;24(suppl 1):57-65.

2. Grubel C, Borovicka J, Schwizer W, Fox M, Hebbard G. Diffuse esophageal spasm. Am J Gastroenterol 2008;103:450-457.

3. Khatami SS, Khandwala F, Shay SS, Vaezi MF. Does diffuse esophageal spasm progress to achalasia? A prospective cohort study. Dig Dis Sci 2005;50:1605-1610. 